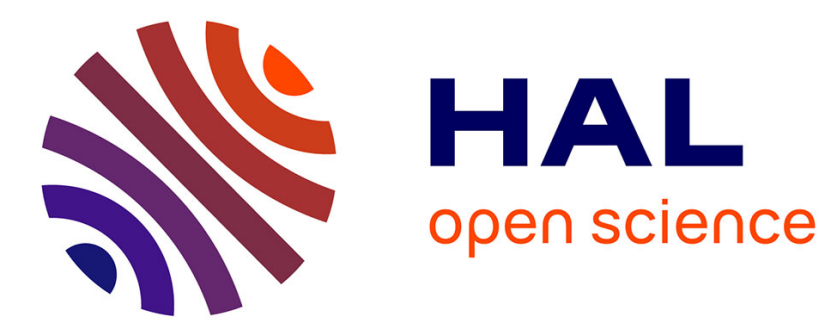

\title{
Construction of real algebraic numbers in Coq
}

Cyril Cohen

\author{
- To cite this version: \\ Cyril Cohen. Construction of real algebraic numbers in Coq. 2012. hal-00671809v1
}

\section{HAL Id: hal-00671809 https://hal.inria.fr/hal-00671809v1}

Preprint submitted on 18 Feb 2012 (v1), last revised 13 Jun 2012 (v2)

HAL is a multi-disciplinary open access archive for the deposit and dissemination of scientific research documents, whether they are published or not. The documents may come from teaching and research institutions in France or abroad, or from public or private research centers.
L'archive ouverte pluridisciplinaire HAL, est destinée au dépôt et à la diffusion de documents scientifiques de niveau recherche, publiés ou non, émanant des établissements d'enseignement et de recherche français ou étrangers, des laboratoires publics ou privés. 


\title{
Construction of real algebraic numbers in $\mathrm{COQ}$
}

\author{
Cyril Cohen
}

February 18, 2012

\begin{abstract}
This paper shows a construction in CoQ of the set of real algebraic numbers, together with a formal proof that this set has a structure of discrete archimedian real closed field. This construction hence implements an interface of real closed field. Instances of such an interface immediately enjoy quantifier elimination thanks to a previous work. This work also intends to be a basis for the construction of complex algebraic numbers and to be a reference implementation for the certification of numerous algorithms relying on algebraic numbers in computer algebra.
\end{abstract}

\section{Introduction}

Real algebraic numbers form the countable subset of real numbers which are roots of polynomials with rational coefficients. This strict sub-field of real numbers has interesting properties that make it an important object for algorithms in computer algebra and in constructive and effective mathematics. Indeed, there is an effective algorithm to compare two algebraic numbers and all field operations can be defined in an exact way. Moreover, they can be equipped with a structure of discrete archimedian real closed field, which is an archimedian ordered field with decidable ordering satisfying the intermediate value property for polynomials.

The aim of this paper is to show how we define in CoQ a data-type representing the real algebraic numbers and to describe how to formally show it has a structure of archimedian real closed field. This construction and these proofs are described in many standard references on constructive mathematics [11] or in computer algebra [2]. However, the implementation of these results in a proof assistant requires various changes in their presentation. Hence our development is not a literate translation of a well-chosen reference, but is rather a synthesis of the bibliography, which chooses the easiest type theoretic formalization for each proof.

In order to define real algebraic numbers, standard references usually suggest one of the following strategies. The first one takes a type representing real numbers and builds the type representing the subset of reals which are roots of a polynomial with rational coefficients. One must then show that induced arithmetic operations on this subset have the expected properties. The second 
strategy starts from a type representing rational numbers and formalizes the real closure of rational numbers, which is the smallest real closed field containing them. An element of the closure is usually represented as pair polynomial interval, satisfying the invariant that the polynomial has a unique root in the interval. This selected root is the algebraic number encoded by that pair. From a constructive point of view, there is no reason to prefer one or the other of these strategies: it may of course be possible to complete the required proofs in any of these two cases. However, there are significant differences in the nature of objects and proofs we handle when formalized in type theory.

In this work, we combine the two approaches in order to get the advantages of both and to eliminate their respective drawbacks.

Constructive formal libraries on exact reals are available in the CoQ system [8]. However, for the requirements of this formalization we developed a short library constructing exact reals as Cauchy sequences from an arbitrary archimedian field. We explain these formalization choices and our construction in section 2 .

Then, in section 3 we introduce a first type for algebraic real numbers which we call algebraic Cauchy reals, together with its comparison algorithm and arithmetic operations. In particular, we show how to compute annihilating polynomials, decide the equality and more generally the comparison.

We then describe in section 4 how to construct the real closure of rational numbers to get a second data-type for real algebraic numbers, that we call real algebraic domain.

Thanks to this second data-type and to the equality decision procedure, we show in section 5 how to form the real algebraic numbers and we prove that it is a real closed field. The key ingredient is the proof of the intermediate value property for polynomials, which concludes this work.

\section{Preliminaries}

In this work, we use the SSREFLECT library of the Mathematical Components project [12]. We base our development on the algebraic hierarchy [7], with the extensions we already brought to describe discrete ordered structures [5]. We

use mostly the discrete real closed field structure. We also take advantage of the available libraries on polynomials with coefficients in rings or fields. More precisely, we use the polynomial arithmetic library which grants the following definitions and properties: arithmetic operations, euclidean division, Bézout theorem, Gauss theorem.

We explain in more details some elements of the SSREFLECT library we use.

In the SSREFLECT library, structures from the algebraic hierarchy include both a decidable equality and a choice operators: they are equipped with equality structures and choice structures. 


\section{Decidable equality structure}

Decidable equality structures are instances of an interface called eqType which provides a boolean relation (eq_op : $\mathrm{T} \rightarrow \mathrm{T} \rightarrow$ bool) which reflects the Leibniz equality, which means:

$\forall(\mathrm{T}:$ eqType $)(\mathrm{x} \mathrm{y}: \mathrm{T}), \mathrm{x}=\mathrm{y} \leftrightarrow$ eq_op $\mathrm{x} \mathrm{y}$

The SSREFLECT library provides a rich theory about eqType, such as for example the uniqueness of equality proofs on such types.

\section{Choice structure}

Choice structures are instances of an interface called choiceType in the library. They provide us the choice operator xchoose of type:

$\underline{\text { xchoose }}: \forall(\mathrm{T}:$ choiceType $)(\mathrm{P}: \mathrm{T} \rightarrow \mathrm{bool}),(\exists \mathrm{x}, \mathrm{P} \mathrm{x}) \rightarrow \mathrm{T}$.

which satisfies the two following properties :

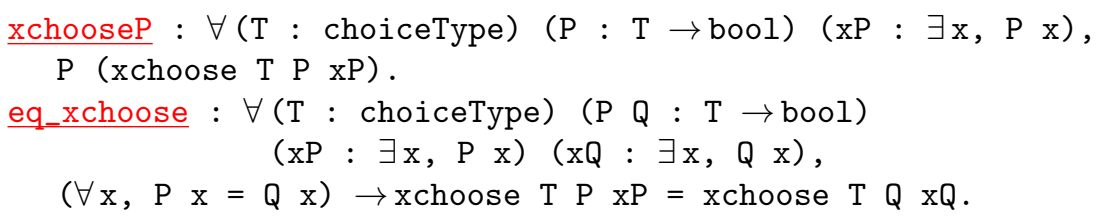

which respectively ensure the correctness and uniqueness of the chosen element with respect to the predicate $\mathrm{P}$.

For instance, in the CoQ system, any countable type can be provably equipped with such a structure. This means we can take $T$ to be the type $\mathbb{Q}$ of rational numbers.

The choice structure is fundamental to formalize both the comparison of Cauchy reals in section 2.2 and the construction of the effective quotient type in section 5 .

\section{Resultant of two polynomials and corollary to Bézout the- orem}

The notion of resultant is well described and studied in numerous books, we invite the reader to look in one of them, for instance in [10]. In our development we use only the two following properties of the resultant $\operatorname{Res}_{X}(P(X, Y), Q(X, Y)) \in$ $F[Y]$ of polynomials $P, Q \in F[X, Y]$ where $F$ is a field.

$$
\begin{gathered}
\exists U, V \in F[X, Y] / \quad \operatorname{Res}_{X}(P, Q)=U P+V Q \\
\operatorname{Res}_{X}(P, Q)=0 \Leftrightarrow P \text { and } Q \text { are not coprime as polynomials in } X
\end{gathered}
$$

which respectively express that the resultant of $P$ and $Q$ is in the ideal generated by $P$ and $Q$, and is zero if and only if $P$ and $Q$ are not coprime as polynomials in $X$ with coefficients in $F[Y]$. 
Moreover we use the following corollary to Bézout theorem: If $P$ and $Q$ are not coprime as polynomials in $X$ with coefficients in $F[Y]$, there exists $U$ and $V$ in $F[X, Y]$ such that $U$ is non zero, $\operatorname{deg}_{X}(U)<\operatorname{deg}(Q)$ and

$$
U(X, Y) P(X, Y)=V(X, Y) Q(X, Y)
$$

\section{Construction and properties of Cauchy reals}

From now on, we denote by $F$ an archimedian field equipped with a decidable equality structure and with a choice structure. All the constructions are done over $F$ which is, for our purpose, an appropriate generalization of $\mathbb{Q}$. Although it is necessary for this construction, we do not detail the use of the archimedian property for the sake of readability.

\subsection{Mathematical description and COQ data-type}

We define a Cauchy real as a sequence $\left(x_{n}\right)_{n \in \mathbb{N}}$ in $F^{\mathbb{N}}$, together with a convergence modulus $m_{x}: F \rightarrow \mathbb{N}$ such that from the index $m_{x}(\varepsilon)$, the distance between any two elements is smaller than $\varepsilon$. This "Cauchy property" is stated as:

$$
\forall \varepsilon \forall i \forall j, m_{x}(\varepsilon) \leq i \wedge m_{x}(\varepsilon) \leq j \Rightarrow\left|x_{i}-x_{j}\right|<\varepsilon
$$

We encode sequences of elements of $F$ as functions from natural numbers to $F$. Hence, we encode Cauchy reals by packaging the sequence $\left(x_{n}\right)_{n}$, the modulus $m_{x}$ and the "Cauchy property":

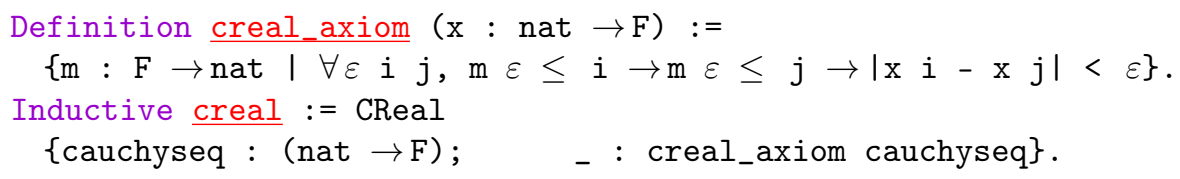

We remind that $\{\mathrm{m}: \mathrm{F} \rightarrow$ nat $\mid \ldots\}$ is called a sigma-type and can be read "there exists a function ( $\mathrm{m}: \mathrm{F} \rightarrow$ nat) such that".

The C-CoRN library also provides an interface for Cauchy reals and a construction of Cauchy sequences, which is used to instantiate the interface in [8]. Although their definition is close enough to ours, we redefine and re-implement Cauchy reals from scratch, mainly because our algebraic structures are incompatible. We use this as an opportunity to restate the definitions in a way which is more compatible with our proof style.

In this paper, we often denote a Cauchy sequence $\left(x_{n}\right)_{n}$ of convergence modulus $m_{x}$ by the notation $\bar{x}$. We call such an element a Cauchy real and it represents a constructive real number. We often take the $i$ th element of the underlying Cauchy sequence of $\bar{x}$, and we note it $x_{i}$. Moreover, in the Coq code, $m_{x}$ is encoded as a function (cauchymod $\mathrm{x}$ ) of type ( $\mathrm{F} \rightarrow$ nat). A CoQ user can remark that such a function is definable because the existential modulus in the definition of the Cauchy sequence is in Type.

By definition of Cauchy sequences, we get the following property: 
Lemma cauchymodP ( $\mathrm{x}: \operatorname{creal})(\varepsilon: F)(\mathrm{i} j:$ nat $)$ :

cauchymod $\mathrm{x} \varepsilon \leq \mathrm{i} \rightarrow$ cauchymod $\mathrm{x} \varepsilon \leq \mathrm{j} \rightarrow|\mathrm{x} i-\mathrm{x}|<\varepsilon$

It is important to note that when we apply this lemma, we produce a subgoal (which we call side condition) of the form $f(\varepsilon) \leq i$. This is a general scheme in our development: during a proof we may generate $n$ side conditions $f_{k}(\varepsilon) \leq i$ for $k \in\{1, \ldots, n\}$. Indeed, if all constraints on $i$ are formulated like this, it suffices to take $i$ to be the maximum of all the $f_{k}(\varepsilon)$, in order to satisfy all the side conditions on $i$. We even have designed an automated procedure to solve this kind of constraints using the Ltac language [6] available in CoQ, so that many proofs begin with a command meaning "let $i$ be a big enough natural number".

From cauchymod we can define a function ubound to bound above the values of elements of a Cauchy sequence. It then satisfies the following property:

Lemma uboundP : $\forall$ ( $\mathrm{x}$ : creal) (i : nat), $|\mathrm{x} i| \leq$ ubound $\mathrm{x}$.

In the rest of the development, this function is used to compute the convergence moduli of numerous Cauchy sequences. We use the notation $\lceil x\rceil$ for (ubound $\mathrm{x})$.

\subsection{Comparison}

On Cauchy reals, the Leibniz equality is not a good notion to compare numbers, as two distinct sequences may represent the same real number. In fact, the good notion of equality on Cauchy reals states that $\bar{x}$ and $\bar{y}$ are equivalent if the sequence of point-wise distances $\left(\left|x_{n}-y_{n}\right|\right)_{n}$ converges to 0 .

A type together with an equivalence relation is called a setoid, and the equivalence is the setoid equality. COQ provides tools to declare setoids, to declare functions that are compatible with the setoid equality, and eventually to rewrite using the setoid equality in contexts that are compatible with it [1].

Although the comparison of Cauchy reals is not decidable, telling whether $\bar{x}$ and $\bar{y}$ are distinct is semidecidable: by looking at $x_{n}$ and $y_{n}$ for a big enough $n$, we may observe that they are distinct, but we can never be sure they are equal. Hence the primitive notion for comparison is not equality but apartness, which contains an additional information: a witness for the non-negative lower bound of the gap separating the two sequences.

For the sake of clarity we write $\bar{x} \neq \bar{y}$ for apartness and $\bar{x} \equiv \bar{y}$ for its negation. The notion of non apartness coincides with the notion of equivalence stated above and is declared as the setoid equality on Cauchy reals

From a proof of apartness $\bar{x} \neq \bar{y}$ we must be able to extract a non-negative witness $\delta$ which bounds below the sequence $\left(\left|x_{n}-y_{n}\right|\right)_{n}$ from some rank $k$. This lower bound is needed to define the inverse as described in section 2.4. So we could define apartness as follows, using a witness in Type to make it available for computation:

Definition bad_neq_creal $\mathrm{x}$ y : Type := $\{\delta: \mathrm{F} \mid 0<\delta \&$

$\forall \mathrm{i}$, cauchymod $\mathrm{x} \delta \leq \mathrm{i} \rightarrow$ cauchymod $\mathrm{y} \delta \leq \mathrm{i} \rightarrow \delta \leq|\mathrm{x} i-\mathrm{y} i|\}$. 
But to be fully compatible with the setoid mechanism, the apartness must be in Prop, not in Type. Russell O'Connor et Robbert Krebbers [9] already encountered this problem in $\mathrm{C}-\mathrm{CoRN}$ and solved it using the "constructive indefinite description" theorem, which is provable for decidable properties which domain is nat. Our solution uses a variant of this theorem, thanks to the choiceType structure of $\mathrm{F}$.

We define apartness $(\neq)$ as follows:

Definition neq_creal ( $\mathrm{x}$ y : creal) : Prop :=

$\exists \delta,(0<\delta)$ \&\& $(3 * \delta \leq \mid \mathrm{x}$ (cauchymod $\mathrm{x} \delta)-\mathrm{y}$ (cauchymod $\mathrm{y} \delta) \mid)$.

Then, using xchoose we can define the non-negative lower bound function:

Definition lbound $\mathrm{x} y($ neq_xy $: \mathrm{x} \neq \mathrm{y}): \mathrm{F}:=\mathrm{xchoose} F$ - neq_xy.

Given two Cauchy reals $\bar{x}$ and $\bar{y}$ which are provably apart from each other, let $\delta$ be their non-negative lower bound of separation as defined above. From $\mathrm{xchooseP}$ we get that

$$
\begin{gathered}
3 \delta \leq\left|x_{m_{x}(\delta)}-y_{m_{y}(\delta)}\right| \\
\text { Thus: } \quad \forall i, j, \quad 3 \delta \leq\left|x_{m_{x}(\delta)}-x_{i}\right|+\left|x_{i}-y_{j}\right|+\left|y_{i}-y_{m_{y}(\delta)}\right|
\end{gathered}
$$

But since we work on Cauchy sequences, we know how to bound the distance between any two elements of the sequence, starting from a well chosen index: $\forall i \geq m_{x}(\delta),\left|x_{m_{x}(\delta)}-x_{i}\right|<\delta$ and $\forall i \geq m_{y}(\delta),\left|y_{i}-y_{m_{y}(\delta)}\right|<\delta$, So

$$
\forall i, j \geq \max \left(m_{x}(\delta), m_{y}(\delta)\right), \quad \delta \leq\left|x_{i}-y_{j}\right|
$$

Hence we prove the lemma:

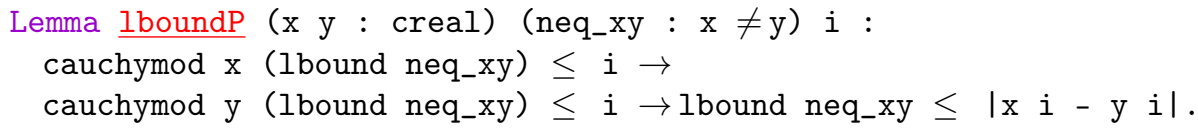

\subsection{Order relation}

The order relation is handled the same way as apartness. The primitive notion is the strict ordering, the negation of which defines the large ordering. In fact, order does not deserve a special treatment as it can be deduced from apartness using the following lemma:

Lemma neq_ltVgt $(x \mathrm{y}:$ creal) $: \mathrm{x} \neq \mathrm{y} \rightarrow\{\mathrm{x}<\mathrm{y}\}+\{\mathrm{y}<\mathrm{x}\}$.

where the operator + is the disjunction in Type. In this paper we often omit the study of the order as the notion is similar to apartness. Indeed, proofs look the same or can be deduced from the apartness.

\subsection{Arithmetic operations on Cauchy reals}

We build the opposite, addition and multiplication on Cauchy reals and prove their output are Cauchy sequence in a systematic way: we perform the appropriate operation on each element of the sequence and we forge a convergence modulus for each operation. 
To build the opposite, addition and multiplication, we exhibit the convergence moduli of opposite, addition and multiplication of Cauchy reals. Given the convergence modulus $m_{x}$ of $\bar{x}$, we prove the convergence moduli of $\left(-x_{n}\right)_{n}$, $\left(x_{n}+y_{n}\right)_{n}$ and $\left(x_{n} y_{n}\right)_{n}$ are respectively: $m_{x}, \varepsilon \mapsto \max \left(m_{x}\left(\frac{\varepsilon}{2}\right), m_{y}\left(\frac{\varepsilon}{2}\right)\right)$ and $\varepsilon \mapsto \max \left(m_{x}\left(\frac{\varepsilon}{2\lceil y\rceil}\right), m_{y}\left(\frac{\varepsilon}{2\lceil y\rceil}\right)\right)$

To build the inverse, we need to know a non-negative lower bound $\delta$ for the sequence $\left(\left|x_{n}\right|\right)_{n}$ of absolute values from some arbitrary rank, and use it to prove that the sequence of point-wise inverses $\left(\frac{1}{x_{n}}\right)_{n}$ is a Cauchy sequence. According to section 2.2, such a non-negative lower bound $\delta$ is given by (lbound $\mathrm{x}_{-}$neq0) as soon as we dispose of a proof ( $\mathrm{x}_{-}$neq0 $: \mathrm{x} \neq 0$ ) that $\bar{x}$ is apart from 0 (in the sense of Cauchy sequences). This value $\delta$ is such that $\forall i>m_{x}(\delta), \delta \leq|x i|$

If $i$ and $j$ are greater than $m_{x}\left(\varepsilon \delta^{2}\right)$, we have

$$
\left|x_{i}-x_{j}\right|<\varepsilon \delta^{2}
$$

By definition of $\delta$ and if $i$ and $j$ are greater than $m_{x}(\delta)$, we get $\delta \leq\left|x_{i}\right|$ and $\delta \leq\left|x_{j}\right|$, thus $\left|x_{j}-x_{i}\right|<\varepsilon\left|x_{i} x_{j}\right|$. And finally

$$
\left|\frac{1}{x_{i}}-\frac{1}{x_{i}}\right|<\varepsilon
$$

Thus, a convergence modulus is $\varepsilon \mapsto \max \left(m_{x}\left(\varepsilon \delta^{2}\right), m_{x}(\delta)\right)$

Morphism property of arithmetic operations. We can check that all arithmetic operations are compatible with the equality for Cauchy sequences, using a simple point-wise study. The order relation is also a compatible.

\subsection{Bounds and evaluation for polynomials}

Now we describe the evaluation of a polynomial of $F[X]$ to a Cauchy real. Once more, we build it as the point-wise operation. However, to prove that the resulting sequence is a Cauchy sequence, we must bound $|P(x)-P(y)|$ when $|x-y|$ is small enough.

We use bounds given by the Taylor expansion of polynomial $P$ that can be computed using coefficients of $P$ and such that for all $x$ and $y$ in the interval $[c-r, c+r]$,

$$
\begin{aligned}
|P(x)| & \leq B_{0}(P, c, r) \\
|P(y)-P(x)| & \leq|y-x| B_{1}(P, c, r) \\
\left|\frac{P(y)-P(x)}{y-x}-P^{\prime}(x)\right| & \leq|y-x| B_{2}(P, c, r)
\end{aligned}
$$

We can now define the evaluation $P(\bar{x})$ of a polynomial in a Cauchy real by the point-wise evaluation $\left(P\left(x_{n}\right)\right)_{n}$ together with the convergence modulus 
$\varepsilon \mapsto m_{x}\left(\frac{\varepsilon}{B_{1}(P, 0,\lceil x\rceil)}\right)$. We then prove that $P(\bar{x}) \neq P(\bar{y}) \Rightarrow \bar{x} \neq \bar{y}$, which implies that $\bar{x} \equiv \bar{y} \Rightarrow P(\bar{x}) \equiv P(\bar{y})$, hence the evaluation of a polynomial in a Cauchy real is compatible with the equality of Cauchy reals.

\section{An existential type for algebraic Cauchy reals}

\subsection{Construction of algebraic Cauchy reals}

Now, we formalize real algebraic numbers on top of Cauchy reals.

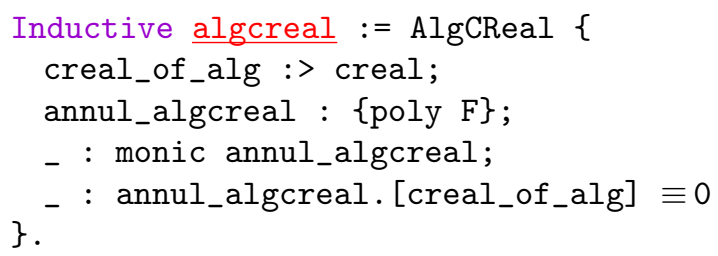

Here, an algebraic Cauchy real (AlgCReal x P monic_P root_P_x) represents an algebraic number as a Cauchy reals $\mathrm{x}$ and a polynomial $\mathrm{P}$ with a proof monic_P that $\mathrm{P}$ is monic and a proof root_P_ $\mathrm{x}$ that $\mathrm{x}$ is a root of $\mathrm{P}$.

First we prove that Cauchy reals setoid equality is decidable on algebraic Cauchy real, then we build arithmetic operations.

\subsection{Equality decision procedure}

Whereas the comparison on Cauchy reals is only semi-decidable, the comparison on algebraic Cauchy reals is decidable. The decision procedure uses the additional data given by the annihilating polynomials. In fact, we only need to decide if some polynomial is zero, because we can test whether $p=q$ by comparing $p-q$ to 0 once we have the subtraction.

Let $(\bar{x}, P)$ be a algebraic Cauchy real we wish to compare to 0 , so $P$ is the annihilating polynomial of the Cauchy real $\bar{x}$. There are two possibilities:

Either $X$ does not divides $P$, then 0 is not a root of $P$, thus $x \neq 0$.

Or $X$ divides $P$. If $P=X$ then $x \equiv 0$, so let us suppose that $X$ is a proper divisor of $P$. Then there exists a divisor $D$ of $P$ whose degree is smaller than the one of $P$ and such that $D(x) \equiv 0$. We can now iterate this reasoning on $(\bar{x}, D)$ where the degree of $D$ is smaller than the one of $P$.

Remark that decidability of order is a direct consequence of this and lemma neq_ltVgt of section 2.3 .

\subsection{Operations on algebraic Cauchy reals}

We build all the operations (opposite, addition, multiplication, inverse) from the constants 0 and 1 and using the subtraction and the division. The embedding 
of the constants $c \in F$ is obtained from the pair $(\bar{c}, X-c)$ (where $\bar{c}$ is a constant Cauchy sequence).

In the remaining of this section we consider two algebraic Cauchy reals $x$ and $y$, whose respective Cauchy sequences are $\bar{x}$ and $\bar{y}$, and whose respective annihilating polynomials are $P$ and $Q$.

Let us recall (section 2.4) that the subtraction $\bar{x}-\bar{y}$ (resp. division $\frac{\bar{x}}{\bar{y}}$ ) is obtained as the point-wise subtraction (resp. division) of elements of the sequence. Let us find a polynomial whose root is this new sequence.

\section{Subtraction}

Our candidate is the resultant

$$
R(Y)=\operatorname{Res}_{X}(P(X+Y), Q(X))
$$

There are two essential properties to prove about this resultant: it is non zero and it annihilates the subtraction.

$R$ is non zero. Let us suppose that $R$ is zero and find a contradiction. Since $R$ is zero, $P(X+Y)$ and $Q(X)$ are not coprime.

Thanks to the corollary to Bézout theorem, we know there exists $U, V \in$ $F[X]$ such that $U$ is non zero, $\operatorname{deg}_{X}(U)<\operatorname{deg}(Q)$ and

$$
U(X, Y) P(X+Y)=V(X, Y) Q(X)
$$

Taking the $Y$-leading coefficient, we get $u(X)=v(X) Q(X)$

where $u(X)$ and $v(X)$ are the respective $Y$-leading coefficients of $U(X, Y)$ and $V(X, Y)$. This equation gives that $\operatorname{deg}(Q) \leq \operatorname{deg}(u), \operatorname{but} \operatorname{deg}(u) \leq \operatorname{deg}_{X}(U)<$ $\operatorname{deg}(Q)$. This is a contradiction.

$R$ annihilates the subtraction. Let us prove that $R$ annihilates the Cauchy sequence $\bar{x}-\bar{y}$. Since $R$ is in the ideal generated by $P(X+Y)$ and $Q(X)$, there exists $U$ and $V$ such that $R(Y)=U(X, Y) P(X+Y)+V(X, Y) Q(X)$. Hence by evaluation at $X=y_{n}$ and $Y=\left(x_{n}-y_{n}\right)$ :

$$
R\left(x_{n}-y_{n}\right)=U\left(y_{n}, x_{n}-y_{n}\right) P\left(x_{n}\right)+U\left(y_{n}, x_{n}-y_{n}\right) Q\left(y_{n}\right)
$$

But $P(\bar{y}) \equiv 0$ and $P(\bar{x}) \equiv 0$. As $y_{n}$ and $x_{n}$ are bounded and $U$ is bounded on a bounded domain (cf section 2.5) we have that $R(\bar{x}-\bar{y}) \equiv 0$.

\section{Division}

When $y$ is zero, we return the annihilating polynomial $X$. When it is non zero, we can find a new $Q$ annihilating $y$ such that $Q(0) \neq 0$. The annihilating polynomial of $\frac{x}{y}$ is the resultant

$$
R(Y)=\operatorname{Res}_{X}(P(X Y), Q(X))
$$


$R$ is non zero. Let us suppose that $R$ is zero and find a contradiction. Since $R$ is zero, $P(X Y)$ and $Q(X)$ are not coprime.

Thanks to the corollary to Bézout theorem, we know there exists $U, V \in$ $F[X]$ such that $U$ is non zero, $\operatorname{deg}_{X}(U)<\operatorname{deg}(Q)$ and

$$
U(X, Y) P(X Y)=V(X, Y) Q(X)
$$

By evaluation at $Y=0$ we get: $U(X, 0) P(0)=V(X, 0) Q(X)$

Since $F[Y]$ is an integral domain, if $V(X, 0)=0$ we know that $Y \mid V(X, Y)$, and that there are two possibilities:

- Either $U(X, 0)=0$, which means $Y \mid U(X, Y)$. Hence, there exists $U^{\prime}(X, Y)$ and $V^{\prime}(X, Y)$, whose degrees in $Y$ are strictly smaller than the ones of $U$ and $V$, and such that:

$$
U^{\prime}(X, Y) P(X Y)=V^{\prime}(X, Y) Q(X)
$$

- Or $P(0)=0$, which means $X \mid P(X)$, thus $X Y \mid P(X Y)$. But we also know that

$$
U(0, Y) P(0)=V(0, Y) Q(0)
$$

And since $Q(0) \neq 0$, we necessarily have $V(0, Y)=0$. It follows that $X \mid V(X, Y)$ and as we already knew that $Y \mid V(X, Y)$, we find that $X Y \mid V(X, Y)$.

Thus, there exists $P^{\prime}$ and $V^{\prime}$ whose degrees are strictly smaller than those of $P$ and $V$ respectively, such that

$$
U(X, Y) P^{\prime}(X Y)=V^{\prime}(X, Y) Q(X)
$$

Hence, we can suppose without loss of generality that none of the members of the following equality cancels.

$$
U(X, 0) P(0)=V(X, 0) Q(X)
$$

This equation gives $\operatorname{deg}(Q) \leq \operatorname{deg}(U(X, 0))$, but we had $\operatorname{deg}(U(X, 0)) \leq \operatorname{deg}_{X}(U)<$ $\operatorname{deg}(Q)$. This is a contradiction.

$R$ annihilates the division. In the same way we did for subtraction, we show that $R\left(\frac{\bar{x}}{\bar{y}}\right) \equiv 0$

\section{Encoding algebraic Cauchy reals}

The data-type of algebraic Cauchy reals is a setoid whose equivalence is decidable, and it is difficult to show that algebraic Cauchy reals form a countable setoid if $F$ is countable. However, we can do better and build a type whose decidable equivalence reflects Leibniz equality, and for which we can exhibit a bijection with $\mathbb{N}$ if $F$ is countable. 
In order to get the type of real algebraic numbers, we should quotient the type of algebraic Cauchy reals by the setoid equality. We know from [3] that this quotient can be done inside COQ as soon as the type which gets quotiented has a choiceType structure and the equivalence relation by which we quotient is decidable. Since algcreal cannot directly be equipped with a choiceType structure, we create a type algdom we call real algebraic domain. The type algdom only serves as an encoding of algcreal in order to forge the quotient, the construction of which we detail in section 5 .

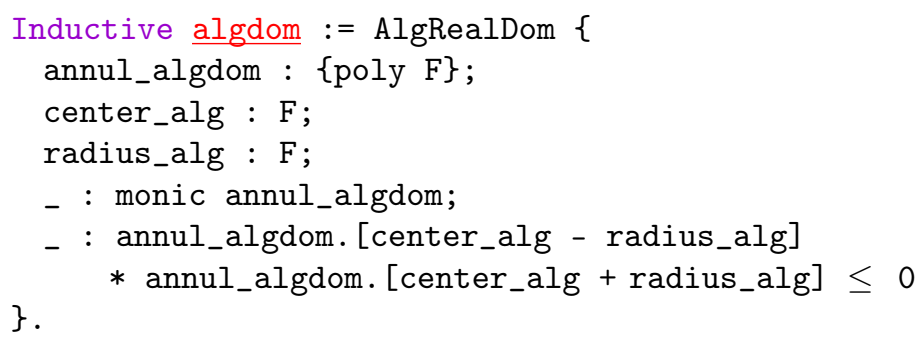

An element (AlgRealDom P c r monic_P chg_sign_P) of algdom represents one of the roots of the polynomial $P$ in the interval $[c-r, c+r]$, with a proof monic_P that $\mathrm{P}$ is monic and a proof chg_sign_P that $\mathrm{P}$ changes sign on the interval. We know which root is selected by running the decoding procedure described in section 4.1.

This data-type is only using elements of $F$ and two proofs. It thus can be encoded as sequences of elements of $F$ and inherits the choiceType structure of $F$. We also notice that algdom is countable as soon as $F$ is. This fact was not obvious for the setoid of algebraic Cauchy reals. The quotient type will also inherit from the choiceType structure and will be countable if $F$ is.

We show that algdom is an explicit encoding of algebraic Cauchy reals.

\subsection{Decoding to algebraic Cauchy reals}

We build the decoding function:

Definition to_algcreal : algdom $\rightarrow$ algcreal

An element from the real algebraic domain contains a polynomials $P$, a center $c$ and a radius $r$ such that $P(c-r) P(c+r) \leq 0$. The root we wish to select is in the interval $I=[c-r, c+r]$.

We decode an element from the real algebraic domain into an algebraic Cauchy real by dichotomy. We form the Cauchy sequence $\bar{x}=\left(x_{n}\right)_{n}$, such that all the $x_{n}$ are in the interval $I$ and such that $P(\bar{x}) \equiv \overline{0}$.

We proceed by induction on $\mathrm{n}$ to define the sequence $\bar{x}$. It should satisfy the following invariant, which expresses that $P$ must change sign on the interval of radius $2^{-n} r$ and centered in $x_{n}$ :

$$
H_{n}=P\left(x_{n}-2^{-n} r\right) P\left(x_{n}+2^{-n} r\right) \leq 0
$$


Base case. We pose $x_{0}=c$. We have $P\left(x_{0}-r\right) P\left(x_{0}+r\right) \leq 0$

Induction step. Suppose we have $x_{n}$ satisfying $H_{n}$. It means

$$
P\left(x_{n}-2^{-n} r\right) P\left(x_{n}+2^{-n} r\right) \leq 0
$$

Thus either $a=x_{n}-2^{-(n+1)} r$ or $b=x_{n}+2^{-(n+1)} r$ satisfy the invariant $H_{n+1}$. Indeed, if it was not the case, we would have:

$$
P\left(a-2^{-(n+1)} r\right) P\left(a+2^{-(n+1)} r\right) P\left(b-2^{-(n+1)} r\right) P\left(b+2^{-(n+1)} r\right) \geq 0
$$

This implies $P\left(x_{n}-2^{n} r\right)\left(P\left(x_{n}\right)\right)^{2} P\left(x_{n}+2^{n} r\right) \geq 0$, which is in contradiction with $H_{n}$. We then chose

$$
x_{n+1}= \begin{cases}a & \text { if } a \text { satisfies } H_{n+1} \\ b & \text { else }\end{cases}
$$

Since $\left|x_{n+1}-x_{n}\right|=2^{-(n+1)} r$, the result is a Cauchy sequence.

The condition that it changes sign is sufficient to show the existence of a root, and doesn't assert anything about its unicity. However, we have no need for unicity as the decoding procedure selects a root in a deterministic manner.

\subsection{Encoding of algebraic Cauchy reals}

This step is more difficult, we construct the encoding function:

Definition to_algdom : algcreal $\rightarrow$ algdom

In order to satisfy the coding property:

Lemma to_algdomK $\mathrm{x}$ : to_algcreal (to_algdom $\mathrm{x}$ ) $\equiv \mathrm{x}$.

Given an algebraic Cauchy real $(\bar{x}, P)$ we try to find a rational interval containing only one root, in order for the decoding to return an element equivalent to $\bar{x}$.

There are two possibilities:

Either $P$ and $P^{\prime}$ are coprime, then there exists $U$ and $V$ such that $U P+$ $V P^{\prime}=1$. Since $P(\bar{x})$ converges to 0 and if $n$ is big enough we get $P^{\prime}\left(x_{n}\right) \geq$ $\frac{1}{2\lceil V(\bar{x})\rceil}$. By taking a small enough interval $[a, b]$ containing $x_{n}$, we get that $P$ is monotone on $[a, b]$ (thanks to the $B_{2}$ bound of section 2.5)

Without loss of generality, we can suppose that $P$ is increasing, we then get $P(a) \leq P\left(x_{i}\right) \leq P(b)$ for all $i \geq n$. But $P\left(x_{i}\right)$ converges to 0 , so $P(a) \leq 0 \leq$ $P(b)$. We found an interval with only one root for $P$.

Or $P$ and $P^{\prime}$ are not coprime, we can find a proper divisor $D$ of $P$ that still annihilate $x$. We fall back to the study of $(\bar{x}, D)$, where the degree of $D$ is strictly smaller that the one of $P$. 


\subsection{Transfering the operations to the encoding}

We can transpose all the operations and properties of algebraic Cauchy reals to its encoding real algebraic domain. More particularly, equality between algebraic Cauchy reals $\equiv$ (which we showed decidable in 3.2 ) gives a decidable equivalence on real algebraic domain, using the following definition:

eq_algdom $x$ y := (eq_algcreal (to_algcreal x) (to_algcreal y))

And for example the addition is defined in the following way:

add_algdom $x$ y :=

to_algdom (add_algcreal (to_algcreal x) (to_algcreal y))

All the properties of these new operators are easily derived from the properties of the original operators.

\section{Real algebraic numbers as a quotient type}

The construction of the quotient is done in a generic way, but for this paper to be self-contained, we inline here its construction as it is automatically done by a mechanism presented in [3].

\subsection{Construction of the quotient type}

First we define a notion of canonical element. To each element $\mathrm{x}$ in algdom, we associate an element (canon $\mathrm{x}$ ) which must be equal to any (canon $\mathrm{y}$ ) if and only if eq_algdom $x \mathrm{y}$. We use the unique choice operator xchoose to do this:

Lemma exists_eq ( $\mathrm{y}:$ algdom) : $\exists \mathrm{x}:$ algdom, $\mathrm{y} \equiv \mathrm{x}$.

Proof. exists y; reflexivity. Qed.

Definition canon $(\mathrm{y}:$ algdom) $=$ xchoose (exists_eq $\mathrm{y}$ ).

For all $\mathrm{x}$ of type algdom, Moreover, canon is constant in each equivalence class thanks to the unicity property of xchoose.

Then we define the quotient type of real algebraic numbers by forming the sigma-type of elements of the real algebraic domain that are canonical:

Definition $\underline{\mathrm{alg}}:=\{\mathrm{x}$ : algdom $\mid$ canon $\mathrm{x}=\mathrm{x}\}$

Thanks to the uniqueness of equality proofs on algdom, two elements $\mathrm{x}$ and $\mathrm{y}$ in alg are equal if and only if ( $\mathrm{val} \mathrm{x}=\mathrm{val} \mathrm{y}$ ), where val is the projection on the first component of the sigma-type. From canon, we can now build the canonical surjection (pi : algdom $\rightarrow$ alg), which maps any element of algdom to its unique equivalence class.

We prove that arithmetic operations (and the order relation) are compatible with the quotient. This is a direct consequence of the morphism property of operations with regard to setoid equality, which we dealt with in section 3.3. 
We also build a function (to_alg: $\mathrm{F} \rightarrow$ alg F) which embeds any element $c$ of $F$ into the equivalence class of the element $(\bar{c},(X-c))$ of algcreal. We then prove it is a field morphism and that this morphism is also compatible with comparison. The mathematical notation for this function is $\uparrow$.

We remark that by construction of algdom, the following property holds: given a polynomial $P \in F[X]$ and two points $a<b \in F$ such that $P(a) \leq 0 \leq$ $P(b)$, there exists $c \in \bar{F}$ such that $c \in[a, b]$ and $P(c)=0$. This is a weak version of the intermediate value property for polynomials, but the general version with $P \in \bar{F}[X]$ and $a, b \in \bar{F}$ is not obvious to prove.

\subsection{Real algebraic numbers form a real closed field}

Now, we prove that $\bar{F}$ is an archimedian totally ordered field with decidable comparison. As those properties already hold for $F$, they transfer to $\bar{F}$ by studying the Cauchy sequences underlying its elements. This means that we can apply the construction of the closure to $\bar{F}$ instead of $F$ and get a "double closure" $\overline{\bar{F}}$.

The difficulty is to prove $\bar{F}$ is a real closed field, which amounts to prove the intermediate value theorem for polynomials in $\bar{F}[X]$.

Let $P$ be a polynomial in $\bar{F}[X]$ and $a$ and $b$ two elements of $\bar{F}$ such that $a<b$ and $P(a) \leq 0 \leq P(b)$. Let us show that there exists a real algebraic number $c$ in $\bar{F}$ such that $P(c)=0$.

\section{Iteration of the closure.}

Thanks to the remark in the end of section 5.1 , by replacing $F$ by $\bar{F}$, we get that the polynomial $P \in \bar{F}[X]$ has a root $\xi \in \overline{\bar{F}}[X]$.

If we find a function $\downarrow: \overline{\bar{F}} \rightarrow \bar{F}$, such that

$$
\forall \zeta \in \overline{\bar{F}}, \quad \uparrow(\downarrow \zeta)=\zeta
$$

then $(\downarrow \xi) \in \bar{F}$ would be a root of $P$. The CoQ name for this function is from_alg. The existence of such a function means that the closure process we design terminates in one step only.

Let $\xi$ be in $\overline{\bar{F}}$, and let us build $(\downarrow \xi)$. By transforming $\xi$ in an algebraic Cauchy real $(\bar{\xi}, P)$ we get a Cauchy sequence $\bar{\xi}$ in $\bar{F}^{\mathbb{N}}$, and a polynomial $P \in$ $\bar{F}[X]$.

Each element $\xi_{n}$ is a Cauchy sequence $\bar{x}_{n}=\left(x_{n, k}\right)_{k}$ which we can chose such that $\left|\bar{x}_{n+1}-\bar{x}_{n}\right|<2^{-(n+1)}$. Then, the sequence $\bar{x}=\left(x_{n, n}\right)_{n}$ is a Cauchy sequence such that $\uparrow \bar{x}=\bar{\xi}$. We hence have the first component of $(\downarrow \xi)$

\section{Polynomial annihilating the algebraic Cauchy real $\bar{x}$.}

We must find a polynomial $R \in F[X]$ which annihilates $\bar{x}$. The coefficients $p_{i}$ of $P$ are a finite number of values in the field extension $\bar{F}$ of $F$, we can apply the primitive element theorem to find an element $\alpha \in \bar{F}$, whose annihilating 
polynomial is $Q$ of degree $q+1$ such that for all $i, p_{i}$ is in the simple extension $F[\alpha]$. We can then re-factorize $P$ as $P=\sum_{l=0}^{q} \alpha^{l} P_{l}$.

We take the resultant

$$
R(Y)=\operatorname{Res}_{X}\left(\sum_{l=0}^{q} X^{l} P_{l}(Y), Q(X)\right)
$$

We now show that it is non zero and it annihilates $\bar{x}$.

$R$ is non zero. Let us suppose $R$ is zero and find a contradiction. The property of Bézout gives $U, V \in F[X]$ such that $U$ is non zero, $\operatorname{deg}_{X}(U)<$ $\operatorname{deg}(Q)$ and

$$
U(X, Y) \sum_{l=0}^{q} X^{l} P_{l}(Y)=V(X, Y) Q(X)
$$

Then by embedding in $\bar{F}$ and evaluation at $X=\alpha$ we get that $U(\alpha, Y) P(Y)=$ 0 . But $P \neq 0$, thus $U(\alpha, Y) P(Y)=0$. Then by taking the $Y$-leading coefficient $u(X)$ of $U(X, Y)$ we get that

$$
u(\alpha)=0 \quad \text { and } \quad u \in F[X] \text { and } u \neq 0 \quad \text { and } \operatorname{deg}(u)<\operatorname{deg}(Q)
$$

This gives a polynomial $u$ annihilating $\alpha$ of degree smaller than the one of $Q$, and we can proceed by induction on the degree of $Q$.

$R$ annihilates $\bar{x}$. We have:

$$
R\left(x_{n, n}\right)=U\left(\alpha_{m}, x_{n, n}\right)\left(\sum_{l=0}^{q} \alpha_{m}^{l} P_{l}\left(x_{n, n}\right)\right)+V\left(\alpha_{m}, x_{n, n}\right) Q\left(\alpha_{m}\right)
$$

and we notice that the right hand side converges to 0 when $m$ and $n$ grow.

\section{Conclusion}

This work provides an instance of the real closed field interface we formalized to deal with its theory [5]. A direct consequence is that real algebraic numbers immediately enjoy quantifier elimination which proves decidable its first order theory. The formalization we describe comes from various classical sources that had to be adapted, made constructive and simplified for the needs of the formalization. The methodology applied here to build algebraic numbers and make proofs feasible and quick is, up to our knowledge, original. This is also, as far as we know, the first certified formalization of real algebraic numbers in a proof assistant.

The complete CoQ formalization we describe in this paper is available at http://perso.crans.org/cohen/work/realalg. Note that, in this paper, we wrote the proofs in a way which is very close to their CoQ formalization. 
It would be interesting to provide an efficient implementation of algebraic numbers, relying on [2] and on [9] for example. The formalization we show in this paper would then serve as a reference implementation. We would need to prove the relative correctness of the efficient implementation with regard to the abstract one. But no proofs about the algebraic structure of the new implementation would be required.

It would be natural to continue this work by extending the real algebraic numbers by the imaginary unit i. Thanks to the constructive fundamental algebra theorem, generalized to real closed fields [4], this new field would be algebraically closed, partially ordered and would then represent the data-type of (complex) algebraic numbers. In the framework of Galois theory, it would also be interesting to formalize the type of algebraic extensions over rational numbers: we could then use the classical presentation and study them into their algebraic closure.

Finally, we formalized the construction of the real closure of fields of zero characteristic, which is a step in constructing the algebraic closure. It is a completely different work to formalize the algebraic closure of fields of non-zero characteristic. Moreover the efficient algorithms for the non-zero characteristic are treated in [2] and are more intricate than the one for the zero characteristic.

\section{Acknowledgement}

I wish to thank Georges Gonthier for the numerous ideas which constitute the basis of this development and Russel O'Connor for discussions which helped me find the good way to state and prove some results. I also thank anonymous french referees for their careful reading and good comments on a draft in french of this paper, and Assia Mahboubi and Enrico Tassi for this version.

\section{References}

[1] Barthe, G., Capretta, V., Pons, O.: Setoids in type theory. J. of Functional Programming 13(2), 261-293 (2003), Special Issue on Logical Frameworks and Metalanguages

[2] Bostan, A.: Algorithmique efficace pour des opérations de base en Calcul formel. Ph.D. thesis, École polytechnique (2003), http://algo.inria.fr/ bostan/these/These.pdf

[3] Cohen, C.: Types quotients en CoQ. In: Hermann (ed.) Actes des 21ème journées francophones des langages applicatifs (JFLA 2010). INRIA, VieuxPort La Ciotat, France (Jan 2010), http://jfla.inria.fr/2010/actes/ PDF/cyrilcohen.pdf

[4] Cohen, C., Coquand, T.: A constructive version of Laplace's proof on the existence of complex roots, http://hal.inria.fr/inria-00592284/PDF/ laplace.pdf, unpublished 
[5] Cohen, C., Mahboubi, A.: Formal proofs in real algebraic geometry: from ordered fields to quantifier elimination, http://hal.inria.fr/ inria-00593738/en/, to appear in Logical Methods in Computer Sciences

[6] Delahaye, D.: A Tactic Language for the System Coq. In: Parigot, M., Voronkov, A. (eds.) Logic for Programming and Automated Reasoning (LPAR). Lecture Notes in Computer Science (LNCS)/Lecture Notes in Artificial Intelligence (LNAI), vol. 1955, pp. 85-95. Springer, Reunion Island (France) (Nov 2000)

[7] Garillot, F., Gonthier, G., Mahboubi, A., Rideau, L.: Packaging mathematical structures. In: Berghofer, S., Nipkow, T., Urban, C., Wenzel, M. (eds.) TPHOLs. Lecture Notes in Computer Science, vol. 5674, pp. 327342. Springer (2009)

[8] Geuvers, H., Niqui, M.: Constructive reals in COQ: Axioms and categoricity. In: Selected papers from the International Workshop on Types for Proofs and Programs. pp. 79-95. TYPES '00, Springer-Verlag, London, UK (2002), http://dl.acm.org/citation. cfm?id=646540.696040

[9] Krebbers, R., Spitters, B.: Computer certified efficient exact reals in COQ. In: Conference on Intelligent Computer Mathematics, CICM 2011 Proceedings. Lecture Notes in Artifical Intelligence, Springer (2011)

[10] Lang, S.: Algebra. Graduate texts in mathematics, Springer (2002)

[11] Mines, R., Richman, F., Ruitenburg, W.: A course in constructive algebra. Universitext (1979), Springer-Verlag (1988)

[12] Project, T.M.C.: SSReflect extension and libraries. http://www. msr-inria.inria.fr/Projects/math-components/index_html 